\title{
Evaluation of the Occluding Characteristics of Nanosized Eggshell/Titanium Dioxide with or without Saliva
}

\author{
Stanley Chibuzor Onwubu ${ }^{1}$ Phumlani Selby Mdluli \\ Mokgadi Ursula Makgobole ${ }^{5}$ \\ ${ }^{1}$ Department of Dental Sciences, Durban University of Technology \\ (DUT), Durban, South Africa \\ 2Department of Chemistry, Durban University of Technology (DUT), \\ Durban, South Africa \\ ${ }^{3}$ Discipline of Dentistry, University of KwaZulu-Natal (UKZN), \\ Westville, South Africa \\ ${ }^{4}$ Microscopy and Microanalysis Unit, University of KwaZulu-Natal \\ (UKZN), Westville, South Africa \\ ${ }^{5}$ Department of Chiropractic and Somatology, Durban University of \\ Technology (DUT), Durban, South Africa
}

Shenuka Singh ${ }^{3}$ Vishal Bharuth ${ }^{4}$

\begin{abstract}
Address for correspondence Stanley Chibuzor Onwubu, MHSc, Dental Sciences Department, Dental Technology Program, Durban University of Technology (DUT), Durban, South Africa (e-mail: 21445599@dut4life.ac.za).
\end{abstract}

Eur J Dent 2019;13:547-555

\begin{abstract}
Keywords

- dentine hypersensitivity

- desensitizing paste

- remineralization

Objectives The study reports on the effectiveness of a ball-milled nanosized titanium dioxide composite ( $\mathrm{EB} @ \mathrm{TiO}_{2}$ ) for $\mathrm{DH}$ management in comparison with commercial desensitizing paste with and without saliva.

Materials and Methods Forty-nine dentine specimens were prepared from extracted bovine anterior teeth. Twenty-one of the specimens were brushed with three desensitizing toothpaste for 7 days, namely: Group 1; ${\mathrm{EB} @ T i O_{2}}_{2}$ Group 2; Colgate Pro-relief; and Group 3; Sensodyne repair $(n=7)$. Twenty-four specimens were brushed with the toothpaste for 7 days and stored in artificial saliva (control) after brushing. Each specimen was subsequently posttreated in citric acid solution to test its stability in acidic condition. Field scanning electron microscope was used to evaluate the effectiveness of the dentine tubules occlusion. The biocompatibility of the composite was tested using BHK21 cell line.

Statistical Analysis One-way analysis of variance was used to analyze the percentage occluded area ratio values for all specimens $(\alpha=0.05)$. Independent $t$-test was further used to evaluate the occlusion differences with saliva and without saliva.

Results and Conclusions The number of dentine tubules decreased significantly after 7 days of brushing. Overall, the occlusion observe for $\mathrm{EB}_{\mathrm{TTiO}}$, were significantly better than for Colgate Pro-relief and Sensodyne repair $(p<0.05)$. BHK21 assay suggested that composite had no significant effect on the BHK21 cell line. This study demonstrated that the composite effectively occluded open dentine tubules within 7 days of brushing.
\end{abstract}

\section{Introduction}

Globalization has created a rapid change in the diets and lifestyles of millions of people worldwide. In South Africa for example, the reintroduction of the country to the global economy postapartheid in 1994 has witnessed the proliferation of foreign goods and a rapidly changing food environment. ${ }^{1}$ Concerning, and in the context of oral health care, 
this change has brought about the increase in consumption of energy drinks, and acidic beverages; which is reportedly linked to the incidence of dental diseases, such as dental caries and erosion of the enamel surface., ${ }^{2,3}$ More worrisome is that the excessive demineralization of the tooth surface due to erosion has been reported to initiate the onset of dentine hypersensitivity (DH). ${ }^{4-6}$

According to the Canadian Advisory Board on $\mathrm{DH},{ }^{7} \mathrm{DH}$ is characterized by distinctive short, sharp pain arising from exposed dentinal tubules particularly in response to external stimuli that are typically thermal, evaporative, tactile, electrical, osmotic, or chemical changes which cannot be ascribed to any other form of dental defects or pathology. As reported in the literature, DH is one of the most clinically encountered problems in dentistry affecting between 10 to $30 \%$ of people worldwide. ${ }^{8}$ Although there have been conflicting reports on the exact prevalence of $\mathrm{DH}$, nevertheless, the most common age range in which $\mathrm{DH}$ is frequently experienced is given as 20 to 50 years, with female patients predominantly affected. ${ }^{9,10}$ Moreover, and as Schiff et al ${ }^{11}$ points out, DH has a negative consequence on the quality of life for dental patient as they are less complaint with oral hygiene recommendation; thus posing a challenge for oral health care providers to manage.

Many theories have been reportedly proposed to explain the mechanism of DH. However, the hydrodynamic theory expanded upon by Brannstrom is now accepted by the dental community as the most likely mechanism for DH occurrence. ${ }^{12}$ In an attempt to control this process, products or agents that typically aimed to reduce fluid flow, and or interfere with the nerve impulses have been reported in the literature. ${ }^{11,13}$ At the first line of at-home therapy for DH management, the use of occluding agents is often recommended for effective treatment. ${ }^{14}$

Several different occluding agents such as potassium oxalates, ${ }^{15}$ sodium fluoride and sodium monofluorophosphate, ${ }^{12}$ strontium salt, ${ }^{16}$ amorphous calcium phosphate containing casein phosphopeptide, ${ }^{17}$ and calcium glycerophosphate ${ }^{18}$ have been widely utilized in desensitizing paste for their dentine tubule occluding capabilities. Still, the effectiveness of the aforementioned occluding agents will depend on the flow of saliva. Moreover, due to the chemical composition of saliva, it can play a critical role in naturally reducing DH. ${ }^{13,19}$ Kleinberg ${ }^{19}$ revealed that saliva could reduce DH by depositing phosphate and calcium ions in the exposed tubules which ultimately result into the ions forming a protective layer on the surface of the tubules. In some patients; however, particularly those with conditions of hyposalivation and xerostomia, the flow of saliva is limited; which could further increase the risks of caries and tooth demineralization, thereby exacerbating $\mathrm{DH}^{20}$

In an attempt to address the above concern, Kleinberg in 2012 at the State University of New York-Stony Brook, patented novel occluding agents based on the understanding of the role that saliva plays in naturally reducing $\mathrm{DH}$. This new technology comprises arginine (an amino acid with a $\mathrm{pH}$ 6.5-7.5), bicarbonate, $\mathrm{pH}$ buffer, and calcium carbonate. ${ }^{19}$ The said technology is marketing under the brand name
Colgate Pro-Argin. ${ }^{11}$ It is reported that Pro-Argin technology function by occluding dentinal tubules using arginine to bind to the negatively charged dentin surface, which subsequently attracts a calcium-rich layer from the saliva to infiltrate and block the dentinal tubules. ${ }^{20}$ However, its effectiveness in a highly acidic environment has been reported to be ineffective, ${ }^{21}$ thus leading to the reopening of the dentine tubules. Given the above drawbacks, a new occluding material consisting of an eggshell modified titanium dioxide composite recently proposed in $\mathrm{DH}$ management. ${ }^{22}$

Importantly, studies ${ }^{23,24}$ have projected that the future of tooth remineralization will be the use of eggshell owing to its high bioavailability of calcium. Likewise, the use of titanium dioxide, particularly in nano form, and their combination with other abrasive agents have been proposed in the literature to occlude open dentine tubules. ${ }^{25}$ In a recent report, the authors demonstrated that eggshell modified with titanium dioxide $\left(\mathrm{EB} @ \mathrm{TiO}_{2}\right)$ significantly improved the composite acidic resistant to erosive acids. ${ }^{26}$ This present study, therefore, aimed to evaluate $\mathrm{EB} @ \mathrm{TiO}_{2}$ occluding characteristic against commercially available toothpaste containing Pro-Argin (Colgate Pro-relief) and NovaMin (Sensodyne repair) with and without saliva in reducing $\mathrm{DH}$. The formulated hypothesis tested was: $\mathrm{EB} @ \mathrm{TiO}_{2}$ significantly occlude the open dentine tubules with or without saliva.

\section{Materials and Methods}

Two commercially available toothpastes namely: Sensodyne repair (GlaxoSmithKline, United Kingdom) and Colgate Pro-relief (Colgate-Palmolive, Poland) were used as the test desensitizing paste. Titanium dioxide (Anatase form) and citric acid were purchased from Sigma-Aldrich (Germany), and Merck (South Africa), respectively.

\section{Eggshell-Titanium Dioxide Composite Preparation}

Eggshell and titanium dioxide composite was prepared in accordance with the method reported in literature. ${ }^{22} \mathrm{An}$ extensive details of the surface morphology, particle sizes, and phase of the prepared $\mathrm{EB} @ \mathrm{TiO}_{2}$ can be found in other reported papers. ${ }^{22,27,28}$

\section{Preparation of Artificial Saliva}

Artificial saliva was prepared following the method reported by Saporeti et a ${ }^{29}$ with a slight modification. As specified in - Table 1, the listed chemicals were prepared in 1L of volumetric flask using deionized water. The $\mathrm{pH}$ of the prepared saliva was given as 6.5 .

Forty-nine anterior teeth extracted from bovine were collected from an abattoir, South Africa. Disinfecting and cleaning of the teeth followed by immersing in $10 \%$ chloroxylenol solution. With the aid of a diamond saw operating at a minimal speed, and cooled with water, the teeth were sectioned below the enamel-dentinal to prepare a dentine specimen having a dimension of $5 \mathrm{~mm} \times 5 \mathrm{~mm} \times 1 \mathrm{~mm}$. A silicon carbide paper with particle size of 600 grits were further used to wet ground the specimens for 60 seconds. Thereafter, the specimens were embedded in a resin (AMT composite, 
Table 1 Composition of the prepared artificial saliva (mg/L)

\begin{tabular}{|l|l|l|}
\hline Chemicals & Concentration $(\mathbf{m g} / \mathbf{L})$ & Mass $(\mathbf{g})$ \\
\hline $\mathrm{NaH}_{2} \mathrm{PO}_{3} \mathrm{H}_{2} \mathrm{O}$ & 780 & 0.078 \\
\hline $\mathrm{NaCl}$ & 500 & 0.05 \\
\hline $\mathrm{KCl}$ & 500 & 0.05 \\
\hline $\mathrm{CaCl}_{2} \mathrm{H}_{2} \mathrm{O}$ & 795 & 0.0795 \\
\hline $\mathrm{NaS}_{9} \mathrm{H}_{2} \mathrm{O}$ & 5 & 0.0005 \\
\hline$\left(\mathrm{NH}_{4}\right)_{2} \mathrm{SO}_{4}$ & 300 & 0.03 \\
\hline $\mathrm{Citric} \mathrm{Acid}$ & 5 & 0.0005 \\
\hline NaHCO & 100 & 0.01 \\
\hline Urea & 1000 & 0.1 \\
\hline
\end{tabular}

Note: Preparation of dentine tooth specimens.

South Africa). The specimens were then soaked in a solution containing $4 \%$ wt. citric acid for 2 minutes to open up the tubules. As described in - Table 2, the specimens were randomly assigned in different experimental groups.

Each specimen from the respective groups were brushed twice daily (morning and evening) with a toothbrush powered with $1.5 \mathrm{v}$ alkaline battery (Oralwise, China) for $1 \mathrm{~min}$ ute and allowed to dry for 30 seconds before rinsing with deionized water. Brushing was performed at room temperature using $100 \mathrm{mg}$ of respective toothpaste. The slurry of EB@ $\mathrm{TiO}_{2}$ was prepared by mixing $100 \mathrm{mg}$ of the powder $/ 200 \mu \mathrm{L}$ of deionized water. After each brushing protocol, the specimens were immersed in saliva or without saliva as described in - Table 2. At the end of the 7-day brushing, the treated specimens were exposed to $4 \% \mathrm{wt}$. citric acid solution for 2 minutes to determine the resistance to acidic challenge, and subsequently rinsed in deionized before blot drying.

Field Scanning Electron Microscope (FESEM; Carl Zeiss) was used to examine the treated specimens after each day of brushing from each respective group. The instrument was operated in controlled environment and scan at $20 \mathrm{kV}$. Prior to FESEM observation, the specimens were dehydrated, sputter coated with electric conductive gold film. Using the captured image of 1500 magnification, a software (ImageJ; National Institute of Health, United States, http://imagej.nih. gov./ij) was used to compute the occluded tubules ratios by dividing the area of the occluded tubules by the total tubules area $(n=7)$. The \% occluded area ratio were counted and used for statistical evaluation.

\section{Biocompatibility Test}

A cytotoxicity assay was performed on the prepared EB@TiO to evaluate its biocompatibility. Before culturing, the sample was dispersed in a solvent (Dimethyl Sulfoxide). The BHK21 hamster kidney cells were grown in the laboratory following the process of culturing normal tissues. ${ }^{22}$ The cell viability were then evaluated using MTS assay. Auranofin was used as a negative control All analyses were tested in duplicate and performed across two plates $(n=6)$.

\section{Statistical Analysis}

One-way analysis of variance (ANOVA) was used to analyze the mean occluded area ratio within the different groups, followed by a Bonferroni test ( $\alpha=0.05$ ). In addition, the independent $t$-test was used to compare the mean occluded area ratio observe for the specimens treated with saliva and without saliva ( $\alpha=0.05$ ). All analysis was performed using statistical software (IBM SPSS Statistics v24; IBM Corp.).

\section{Results}

Dentine Specimens Treated in 7 Days (without Saliva)

- Table 3 depicts the results of the dentine specimens measured in 7 days without saliva immersion. The total mean \% ratio of the tubules occluded area for the dentine specimens

Table 2 The distribution of specimens according to the experimental group

\begin{tabular}{|c|c|c|c|c|}
\hline \multirow[t]{2}{*}{ Sample groups } & \multicolumn{2}{|c|}{ Treatment condition } & \multirow[t]{2}{*}{ Brushing days } & \multirow[t]{2}{*}{ Tota } \\
\hline & Without saliva & With saliva & & \\
\hline Artificial saliva & - & 7 & \multirow[t]{5}{*}{ Twice daily (for 7 days) } & 7 \\
\hline $\mathrm{EB}_{\mathrm{N}} @ \mathrm{TiO}_{2}$ & 7 & 7 & & 14 \\
\hline Colgate Pro-relief & 7 & 7 & & 14 \\
\hline Sensodyne repair & 7 & 7 & & 14 \\
\hline Total & 21 & 28 & & 49 \\
\hline
\end{tabular}

Note: Surface examination of the treated specimens. EB@TiO ${ }_{2}$, eggshell-titanium dioxide.

Table 3 ANOVA test comparison of the occluded area (without saliva)

\begin{tabular}{|c|c|c|c|c|c|c|c|}
\hline \multirow[t]{2}{*}{ Treatment group } & \multirow[t]{2}{*}{$N$} & \multirow[t]{2}{*}{ Mean \pm SD } & \multirow{2}{*}{$\begin{array}{l}\text { Standard } \\
\text { error }\end{array}$} & \multicolumn{2}{|c|}{$95 \%$ confidence interval } & \multirow[t]{2}{*}{$p$-Value } & \multirow{2}{*}{$\begin{array}{l}\text { Posthoc } \\
\text { Bonferroni's test } \\
p \text {-Value }\end{array}$} \\
\hline & & & & Lower bound & Upper bound & & \\
\hline $\mathrm{EB} @ \mathrm{TiO}_{2}$ & 7 & $64.7 \pm 1.3$ & 0.655 & 63.318 & 66.070 & \multirow[t]{3}{*}{0.000} & $0.028^{1,2}$ \\
\hline Colgate Pro-relief & 7 & $62.0 \pm 3.2$ & 0.655 & 60.624 & 63.376 & & $0.000^{1,3}$ \\
\hline Sensodyne repair & 7 & $22.7 \pm 4.8$ & 0.655 & 21.358 & 24.111 & & $0.000^{2,3}$ \\
\hline
\end{tabular}

Abbreviation: SD, standard deviation.

Note: Superscript numbers indicate significant differences between the sample groups (ANOVA, $p<0.05$ ). EB@TiO ${ }_{2}$, eggshell-titanium dioxide. 
treated with $\mathrm{EB} @ \mathrm{TiO}_{2}$, Colgate Pro-relief, and Sensodyne repair was statistically different $(p<0.001)$.

Notably, and after 7 days of brushing, the $\mathrm{EB} @ \mathrm{TiO}_{2}$ group had the highest \% mean occluded area (64.7 $\pm 1.3 \%)$, while the Sensodyne repair treated group had the lowest \% mean occluded area $(22.7 \pm 4.8 \%)$. The Bonferroni's correction results are given in - Table 3 . The \% tubules occluded for the test group $\left(\mathrm{EB} @ \mathrm{TiO}_{2}\right)$ were statistically higher when compared against the Colgate Pro-relief group $(p<0.05)$, and the Sensodyne repair group $(p<0.001)$. Equally, the \% occluded area measured for the Colgate Pro-relief was significantly higher than that observed for Sensodyne repair $(p<0.001)$. -Fig. 1 illustrates the differences in the \% tubules occluded per day with the three desensitizing paste materials (EB@ $\mathrm{TiO}_{2}$, Colgate Pro-relief, and Sensodyne).

The Paired sample test, mean, and standard deviation results for the dentine specimen's pre- and postacidic challenge are given in $\boldsymbol{- T a b l e ~} \mathbf{4}$. There was no significant different found in the $\mathrm{EB}_{0} \mathrm{TiO}_{2}$ group pre- and postacidic treatment $(p>0.05)$. In contrast, both the Colgate Pro-relief and Sensodyne repair treated group showed differences $(p<0.001)$.

The FESEM image of the occluded dentine tubules for dentine specimens treated without storing in artificial saliva after 7 days brushing test is reflected in - Fig. 2. In day 1 (-Fig. 2B1), day 2 ( - Fig. 2B2), the group treated with Colgate Pro-relief showed more evidence of tubule occlusion when compared against the group treated with EB@ $\mathrm{TiO}_{2}$ and Sensodyne repair. However, the $\mathrm{EB} @ \mathrm{TiO}_{2}$ group showed a better evidence of tubule remineralization in day 3 (-Fig. 2A3) and day 4 (-Fig. 2A4). Similarly, there was a

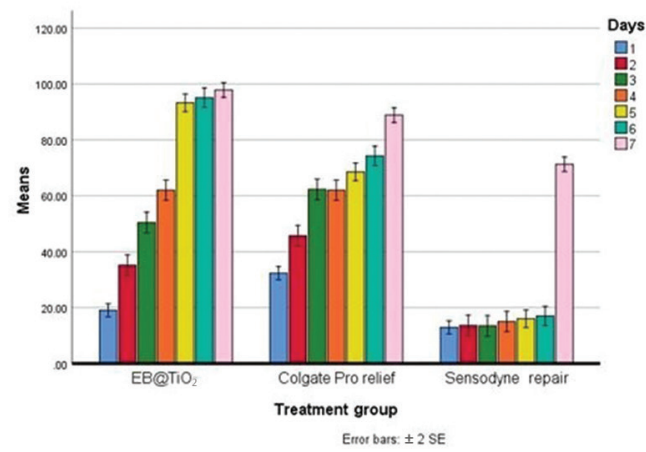

Fig. 1 Differences in mean tubules occluded of dentine specimens treated with EB@TiO, Colgate Pro-relief, and Sensodyne repair desensitizing paste materials after 2 minutes of brushing without saliva immersion (7-day brushing test $[n=7])$. EB@ $@ \mathrm{TiO}_{2}$, eggshell-titanium dioxide. complete remineralization or sealing of the tubules in the $\mathrm{EB} @ \mathrm{TiO}_{2}$ group in day 5 ( - Fig. 2A5), day 6 ( - Fig. 2A6), and day 7 (-Fig. 2A7).

The posttreatment in citric acid solution (4 wt.\%) of the specimens are shown in -Fig. $\mathbf{2}$ (A-C8). Nonetheless, the

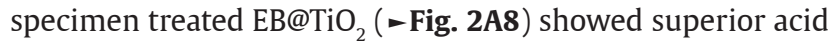
resistance with no visible differences pre- and postacidic challenge when compared against the specimens treated with Colgate Pro-relief ( - Fig. 2B8) and Sensodyne repair ( - Fig. 2C8), respectively.

\section{Dentine Specimens Treated in 7 Days (with Saliva)}

The mean, standard error, standard deviation, and ANOVA results for the dentine specimens stored in artificial saliva after brushing treatment are shown in - Table $\mathbf{5}$. The total mean \% ratio of the tubules occluded area for the dentine specimens stored in artificial saliva alone, treated with

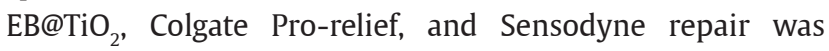
statistically different $(p<0.001)$.

It was found that the \% occluded mean measured for the $\mathrm{EB} @ \mathrm{TiO}_{2}$ group was the highest $(72.0 \pm 1.0 \%)$, while the specimens stored in artificial saliva alone without treatment had the lowest \% mean occluded area $(7.3 \pm 2.3 \%)$. The Bonfer-

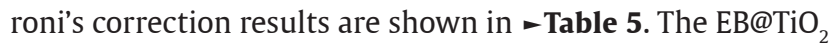
group mean \% occluded area was statistically better when compared against the Colgate Pro-relief, and the Sensodyne repair $(p<0.001)$. More so, the \% occluded area measured for the Sensodyne repair was significantly higher than that observed for Colgate Pro-relief $(p<0.001)$. All the treatment groups showed a significant improvement in occluding the tubules when compared against the samples stored in artificial saliva alone $(<0.001)$. - Fig. 3 illustrates the differences in the \% tubules occluded per day with the three desensitizing paste materials ( $\mathrm{EB} @ \mathrm{TiO}_{2}$, Colgate Pro-relief, and Sensodyne) and artificial saliva.

- Table 6 provides the paired sample test, mean, and standard deviation results for the dentine specimen's (stored in artificial saliva) pre- and postacidic challenge. No difference was found in the Sensodyne repair group pre- and postacidic treatment $(p>0.05)$. By contrast, there was a significant difference observed for the $\mathrm{EB} @ \mathrm{TiO}_{2}$, Colgate Pro-relief, and specimens stored in artificial saliva alone $(p<0.001)$.

The FESEM images of the dentine specimens treated with $\mathrm{EB}^{-\mathrm{TiO}_{2}}$, Colgate Pro-relief, and Sensodyne repair for 7 days and storing in artificial saliva are shown in - Fig. 4. The observed images indicate the occlusion of $\mathrm{EB}-\mathrm{TiO}_{2}$ groups (A1-A7) were different from other test groups (Artificial saliva, Colgate Pro-relief, and Sensodyne repair). - Fig. 4 (A-D8)

Table 4 Paired sample test comparison of occluded area ratio pre- and postacidic treatment

\begin{tabular}{|l|l|l|l|}
\hline \multirow{2}{*}{ Treatment group } & \multicolumn{2}{|c|}{ Occluded area (\%) } & \multirow{2}{*}{$p$-Value } \\
\cline { 2 - 4 } & Preacidic challenge (mean \pm SD) & Postacidic challenge (mean \pm SD) & 0.318 \\
\hline EB@TiO $_{2}$ & $97.9 \pm 1.3$ & $97.1 \pm 1.2$ & 0.000 \\
\hline Colgate Pro-relief & $88.9 \pm 3.2$ & $33.9 \pm 4.1$ & 0.000 \\
\hline Sensodyne repair & $71.3 \pm 4.9$ & $9.3 \pm 2.4$ & \\
\hline
\end{tabular}

Abbreviation: SD, standard deviation. 

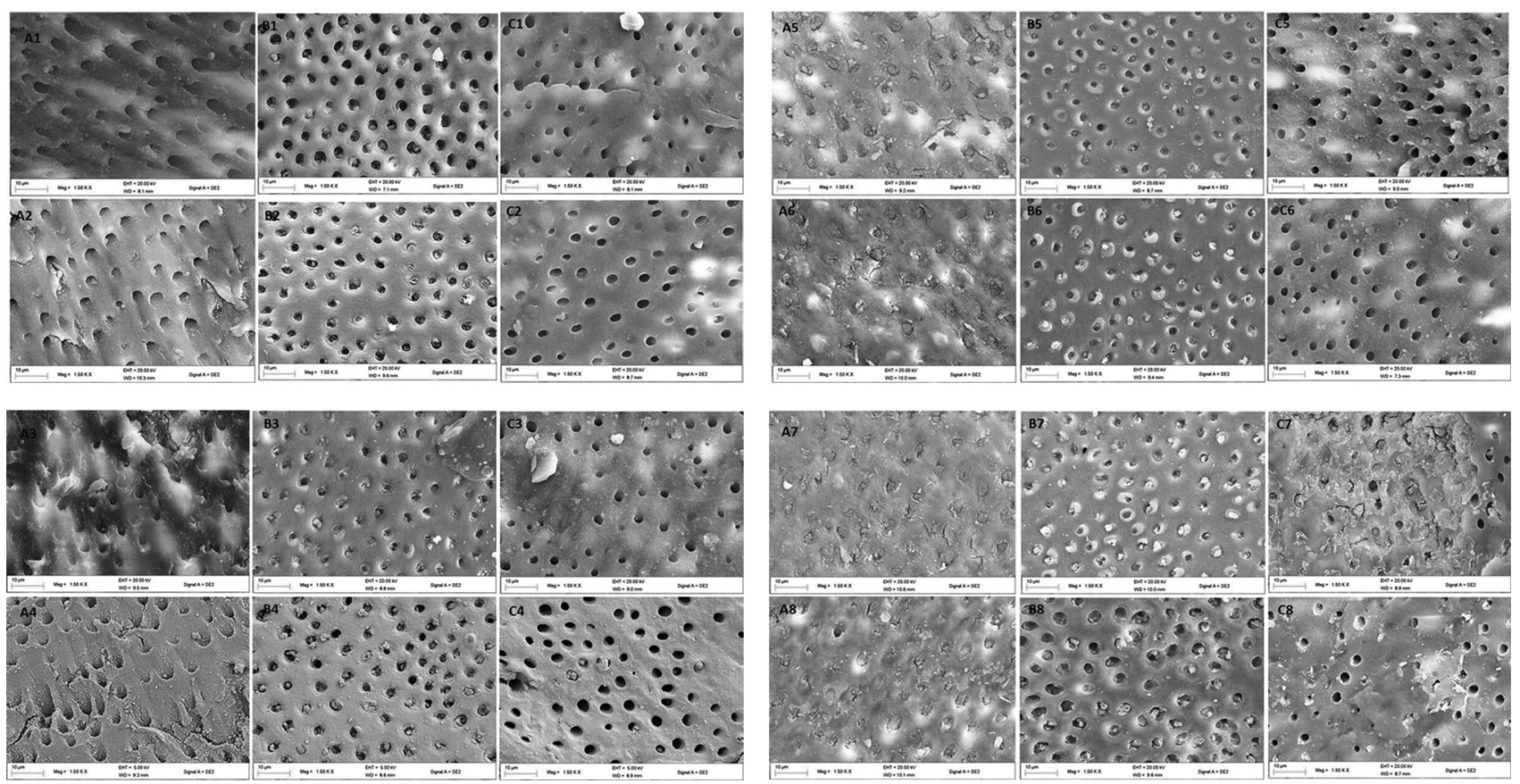

Fig. 2 RepresentativeFESEM micrograph for the dentine surface after brushing for 7 days without saliva immersion using (A)EB@TiO ; (B) Colgate Pro-relief; (C) Sensodyne repair (1-7 represents number of each day of brushing with the respective desensitizing paste, and 8 represent post acidic exposure). FESEM, field scanning electron microscope. EB@TiO ${ }_{2}$, eggshell-titanium dioxide.

Table 5 ANOVA test Comparison of the occluded area (samples stored in artificial saliva)

\begin{tabular}{|c|c|c|c|c|c|c|c|}
\hline \multirow[t]{2}{*}{ Treatment group } & \multirow[t]{2}{*}{$N$} & \multirow[t]{2}{*}{ Mean \pm SD } & \multirow{2}{*}{$\begin{array}{l}\text { Standard } \\
\text { error }\end{array}$} & \multicolumn{2}{|c|}{$95 \%$ confidence interval } & \multirow[t]{2}{*}{$p$-Value } & \multirow{2}{*}{$\begin{array}{l}\text { Posthoc } \\
\text { Bonferroni test } \\
p \text {-Value }\end{array}$} \\
\hline & & & & Lower bound & Upper bound & & \\
\hline Artificial saliva & & $7.3 \pm 2.3$ & 0.636 & 5.953 & 8.578 & \multirow[t]{4}{*}{0.000} & $0.000^{1-5}$ \\
\hline $\mathrm{EB} @ \mathrm{TiO}_{2}$ & & $72.0 \pm 1.0$ & 0.636 & 70.708 & 73.333 & & $0.000^{2,3}$ \\
\hline Colgate Pro-relief & & $34.3 \pm 8.6$ & 0.636 & 33.034 & 35.659 & & $0.000^{3,4}$ \\
\hline Sensodyne repair & & $50.3 \pm 3.0$ & 0.636 & 49.014 & 51.639 & & $0.000^{2,4}$ \\
\hline
\end{tabular}

Abbreviation: SD, standard deviation.

Note: Superscript numbers indicate significant differences between the sample groups (ANOVA, $p<0.001$ ). EB@TiO ${ }_{2}$, eggshell-titanium dioxide.

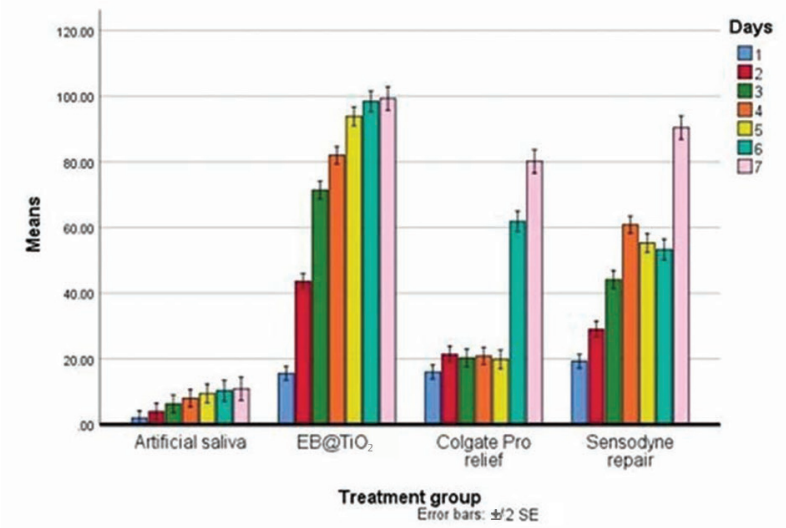

Fig. 3 Differences in mean tubules occluded of dentine specimens treated with $\mathrm{EB} @ \mathrm{TiO}_{2}$ Colgate Pro-relief, and Sensodyne repair desensitizing paste materials after 2 minutes of brushing and immersed in saliva (7 day brushing test $[n=7])$. EB@TiO ${ }_{2}$, eggshell-titanium dioxide. revealed dissimilarity posttreatment of the specimens in citric acid solution (4 wt.\%). The occluded tubules remain intact after acidic challenge for both the EB@TiO 2 and Sensodyne treated group. On the contrary, the tubules in the Colgate Pro-relief ( $\boldsymbol{- \text { Fig. }}$ 4C8) treated specimens were visibly reopened postacidic challenge.

\section{Biocompatibility Testing}

The biocompatibility of $\mathrm{EB} @ \mathrm{TiO}_{2}$ with the BHK21 cell line is shown in - Fig. 5. In comparison to the negative control, the $\mathrm{EB} @ T i O_{2}$ appear to show little effect on the BHK21 cell lines. However, there was $56 \%$ cell viability at $100 \mu \mathrm{g} / \mathrm{mL}$.

\section{Discussion}

Over the last decade, DH has been extensively researched owing to its widespread prevalence and noticeable painful oral health problem affecting many individuals. ${ }^{30}$ The main aim of the paper to evaluate the effectiveness of a modified 
Table 6 Paired sample test comparison of occluded area ratio pre- and postacidic treatment (samples stored in artificial saliva)

\begin{tabular}{|l|l|l|l|}
\hline \multirow{2}{*}{ Treatment Group } & \multicolumn{2}{|c|}{ Occluded area (\%) } & \multirow{2}{*}{$p$-Value } \\
\cline { 2 - 4 } & Preacidic challenge (mean \pm SD) & Postacidic challenge (mean \pm SD) & 0.001 \\
\hline Artificial saliva & $10.9 \pm 2.3$ & $5.7 \pm 1.8$ & 0.000 \\
\hline EB@TiO 2 & $99.3 \pm 1.0$ & $85.0 \pm 3.8$ & 0.000 \\
\hline Colgate Pro-relief & $80.1 \pm 8.6$ & $9.0 \pm 2.2$ & 0.245 \\
\hline Sensodyne repair & $90.4 \pm 3.0$ & $88.1 \pm 3.9$ & \\
\hline
\end{tabular}

Abbreviation: SD, standard deviation.
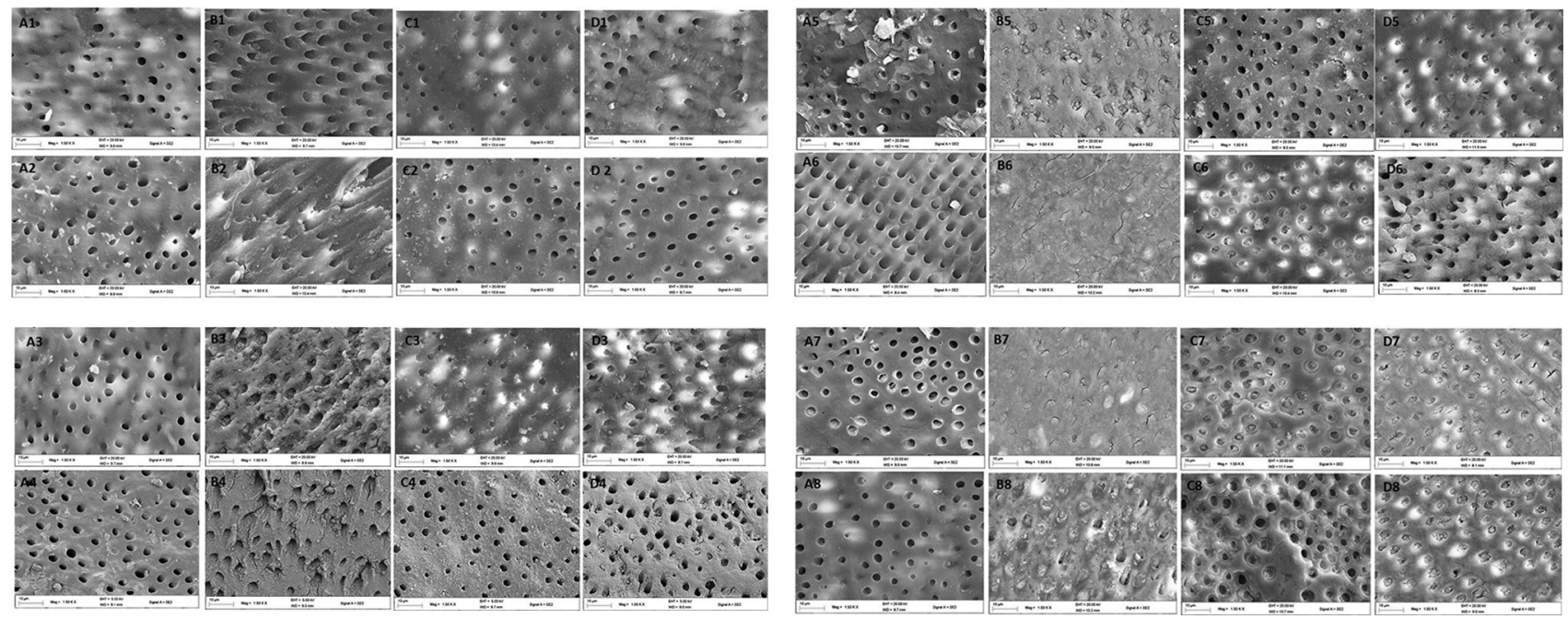

Fig. 4 Representative FESEM micrograph for the dentine surface after brushing for seven days with saliva immersion using (A) Artificial saliva; (B) EB@TiO ; (C) Colgate Pro-relief; (D) Sensodyne repair (1-7 represents with the respective desensitizing paste, and 8 post acidic exposure). FESEM, field scanning electron microscope. EB@TiO ${ }_{2}$, eggshell-titanium dioxide.

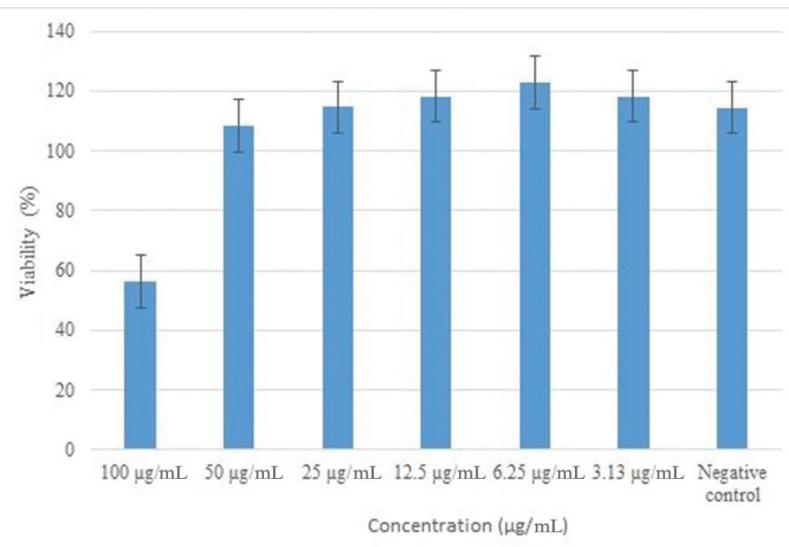

Fig. 5 Percentage cell viability.

nanosized eggshell powder titanium dioxide composite(EB@ $\mathrm{TiO}_{2}$ ) in reducing $\mathrm{DH}$. The composite was prepared through the mechanochemical activation method. Importantly, this method utilizes a mechanical energy to create structural changes as well as stimulate chemical reactions. ${ }^{31}$ Consequently, it becomes possible to cause structural changes and

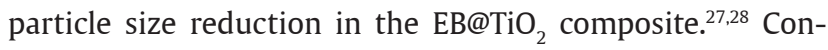

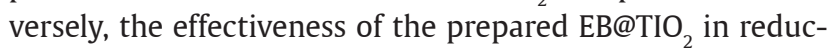
ing DH was compared against Colgate Pro-relief and Sensodyne with or without immersion saliva. As suggested in the literature, ${ }^{32-37}$ their occluding capabilities were evaluated using the bovine model. The morphological changes pre- and postacidic treatment of the specimens were evaluated with FESEM. The EB@TiO 2 treated specimens showed good tubule occlusion that still remain effective in acidic condition for both samples treated with and without saliva. This leads to the acceptance of the study hypothesis.

With respect to time, in the specimens treated without saliva, Colgate Pro-relief showed instant occluding of the dentine tubules. This is consistent with clinical studies $^{38,39}$ that Pro-Argin technology provides instant relief of DH. According to the mechanism proposed by Kleinberg, ${ }^{19}$ it may be assumed that dentine with a negative charge surface attracts the positive charge arginine constituent of the Colgate Pro-relief which subsequently causes the adherence of calcium carbonate to the dentin surface. This in turn promotes the occlusion of the tubules. Despite this, the overall dentine tubule occlusion observed in the samples treated with $\mathrm{EB} @ \mathrm{TiO}_{2}$ were significantly better than Colgate Pro-relief $(p<0.05)$ and Sensodyne repair $(p<0.001)$, respectively. These differences could be attributed to the modification of the carbonate structure in eggshell with titanium dioxide.22 According to Cutler, ${ }^{25}$ nanosized titanium dioxide, together with abrasive materials facilitate the occluding of dentine tubules, thus contributing to reducing of DH. Added to this, the nanosized calcium carbonate materials have unique high surface energy-thus facilitating the attachment of calcium-rich ions on the oral tooth surface. ${ }^{40}$ 
Furthermore, the occlusion measured for Sensodyne repair was statistically lower compared against the Colgate Pro-relief $(p<0.001)$. At the end of 7 days brushing, EB@TiO 2 had the highest occluded area $(97.8 \pm 1.3 \%)$ followed by Colgate Pro-relief ( $88.9 \pm 3.2 \%)$, and lastly Sensodyne repair (71.3 $\pm 4.9 \%)$. Overall, the highest (64.7 \pm 1.3 ) occlusion measured was in the EB@TiO 2 group, while Sensodyne repair had the lowest $(22.7 \pm 4.8$; - Table 3$)$. This difference may be related to the constituent of the various test materials. Although studies ${ }^{41,42}$ have shown that calcium sodium phosphosilicate (NovaMin) constituent of the Sensodyne repair could obstruct dentine tubules to some extent, Yu et al ${ }^{43}$ however, argued that the $\mathrm{Ca}^{2+}$ and $\mathrm{PO}_{4}$ are protected by glass particles which need to be trapped for the $\mathrm{Ca}^{2+}$ and $\mathrm{PO}_{4}$ to be localized. The consequence of this is that there might be a delay in the action of the NovaMin to effectively promote the closing of dentine tubules. ${ }^{43}$ Since the brushing test was performed in 7 days, without saliva, the inferior occluding characteristics observe for Sensodyne repair could be attributed to the absence of saliva to trap the $\mathrm{Ca}^{2+}$ and $\mathrm{PO}_{4}{ }^{3-}$.

On the other hand, for the specimens treated with saliva immersion, all the tested material demonstrated a significant occlusion difference when compared with the those found in saliva alone $(p<0.001)$. While saliva is reported to facilitate remineralization by the deposition of calcium and phosphate, ${ }^{13,19}$ the finding from this study suggests that the occlusion of specimens in saliva alone without desensitizing paste treatment were highly inferior ( - Table 5 ). This may; however, be attributed to the treatment duration ( - Table 2 ). As reported in literature, ${ }^{12}$ the occluding capabilities of saliva occur gradually within a long time. In support of the role saliva plays in reducing $\mathrm{DH}$, the dentine tubules occlusion observed for Sensodyne repair showed an outstanding occlusion when compared against the samples treated without saliva. Similar significant occluding abilities were measured for $\mathrm{EB} @ \mathrm{TiO}_{2}$ treated with saliva immersion $(p<0.05)$.

Contrary to the above, the dentine tubules occlusion observed for the samples treated with Colgate Pro-relief with saliva treatment were consistently inferior at each day of brushing to those measured for the samples treated without saliva $(p<0.001$; - Table 6$)$. In contrast, other studies ${ }^{11,38}$ claimed that the interaction of calcium carbonate and arginine encourages endogenous calcium and phosphate ions to deposit and occlude the dentin tubules. However, Yang et $\mathrm{al}^{44}$ found that Colgate Pro-relief showed no significant changes after treatment and immersion in artificial saliva for 14 days. The above author findings corroborate with the same observation found in this study.

Moreover, the stability of occluding agents, particularly in a high acidic oral environment, is an important criterion for evaluating the efficiency of desensitizing paste in occluding dentine tubules. ${ }^{45}$ This is more important as the oral cavity is often bombarded with citric acid that is highly common in the soft drinks and fruit juices found in our daily diets. In light of these, the effectiveness of the dentine occlusion observed with the different desensitizing paste was assessed posttreatment in a solution containing $4 \mathrm{wt} . \%$ citric acid. The results observed for Colgate Pro-relief suggests that the product demonstrated an acid resistant to a certain extent. This can further be supported by the FESEM images that visibly showed that some of the closed dentine tubules were reopened after exposure to the citric acid solution ( - Fig. 2B8, and 4C8). This; however, could be attributed to the solubility of calcium phosphates in an acidic environment. ${ }^{21}$

In terms of the Sensodyne repair, the acid resistance effectiveness measured exhibit different behavior in the samples treated with and without saliva. In the group treated without saliva, nearly all the tubules were reopened after the exposure to citric acid (-Fig. 2C8). Similar findings were observed by Yu et al $^{43}$ where the deposits created by NovaMin on the dentine surface were almost completely removed by the citric acid solution. In contrast, the samples treated with saliva (-Fig. 4D8), the Sensodyne repair demonstrated an outstanding acidic resistance characteristic $(p>0.05)$. The difference observed for both sample treatments may be associated with the role the saliva plays. It can, therefore, assume that the occlusion for the samples treated with Sensodyne and immersed in saliva had depth and penetration, thereby contributing to its acidic resistance.

As for the $\mathrm{EB} @ \mathrm{TiO}_{2}$ group, the acidic resistant properties observed for the samples treated without saliva, pre- and postcitric acid exposure were comparable $(p>0.05)$. However, slight differences were observed for the samples treated and immersed in saliva. It was found that after citric exposure, some of the obstructing tubules were reopened (-Fig. 4B8). This notwithstanding, the FESEM images visibly validate that the acid resistant characteristics of $\mathrm{EB} @ \mathrm{TiO}_{2}$ were superior to that of Colgate Pro-relief and to some extent Sensodyne repair. Consistent with Tao et $\mathrm{al}^{46}{ }^{46}$ the stability of $\mathrm{EB} @ \mathrm{TiO}_{2}$ in an acidic condition may have been influenced by the modification of eggshell with titanium dioxide. Further clinical research is; however, needed to substantiate the efficiency of EB@TiO 2 as biocomposite material for the management of $\mathrm{DH}$.

\section{Conclusion}

In conclusion, and within the study limitation, the study

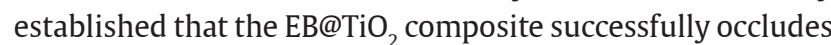
open dentine tubules with and without saliva. It was also established that EB@TiO 2 achieved effectiveness after 3 days of brushing. The composites also provide outstanding acid resistant stability. Despite this, and given the size of the sample used for the study, larger and longer duration of treatment would be required to conclusively determine the efficiency of $\mathrm{EB}_{\mathrm{TiO}}$ in reducing $\mathrm{DH}$.

\section{Funding}

The financial support provided by the National Research Foundation of South Africa (No. 104824) is acknowledged by the authors.

\section{Conflict of Interest}

None declared. 


\section{References}

1 Igumbor EU, Sanders D, Puoane TR, et al. "Big food," the consumer food environment, health, and the policy response in South Africa. PLoS Med 2012;9(7):e1001253

2 Pinto SC, Bandeca MC, Silva CN, Cavassim R, Borges AH, Sampaio JE. Erosive potential of energy drinks on the dentine surface. BMC Res Notes 2013;6:67

3 Giacaman RA, Pailahual V, Díaz-Garrido N. Cariogenicity induced by commercial carbonated beverages in an experimental biofilm-caries model. Eur J Dent 2018;12(1):27-35

4 Salahi S, Ghanbari M, Moosaali F. Effect of Three Common Desensitizers in Reduction of the Dentin Hypersensitivity after Periodontal Surgery. J Dent Biomater. 2016;3:169-176.

5 Rahardjo A, Nasia AA, Adiatman M, Maharani D. Efficacy of a toothpaste containing $5 \%$ potassium nitrate in desensitizing dentin hypersensitivity. Asian J Pharm Clin Res. 2016;9:345-347.

6 Mafla AC, Lopez-Moncayo LF. Dentine sensitivity risk factors: a case-control study. Eur J Dent 2016;10(1):1-6

7 Canadian Advisory Board on Dentin Hypersensitivity. Consensus-based recommendations for the diagnosis and management of dentin hypersensitivity. J Can Dent Assoc 2003;69(4):221-226

8 Clark D, Levin L. Non-surgical management of tooth hypersensitivity. Int Dent J 2016;66(5):249-256

9 Miglani S, Aggarwal V, Ahuja B. Dentin hypersensitivity: recent trends in management. J Conserv Dent 2010;13(4):218-224

10 Colak H, Demirer S, Hamidi M, Uzgur R, Köseoğlu S. Prevalence of dentine hypersensitivity among adult patients attending a dental hospital clinic in Turkey. West Indian Med J 2012;61(2):174-179

11 Schiff T, Delgado E, Zhang YP, Cummins D, DeVizio W, Mateo LR. Clinical evaluation of the efficacy of an in-office desensitizing paste containing $8 \%$ arginine and calcium carbonate in providing instant and lasting relief of dentin hypersensitivity. Am J Dent 200922 Spec No A:8A-15A

12 Merh A, Singhbal K, Parikh V, Mehta S, Kulkarni G. Comparative evaluation of immediate efficacy of diode laser versus desensitizing paste containing $8 \%$ arginine and calcium carbonate in treatment of dentine hypersensitivity: an in vivo study. J Evol Med Dent Sci. 2015;4(25):4346-4355

13 Panagakos F, Schiff T, Guignon A. Dentin hypersensitivity: effective treatment with an in-office desensitizing paste containing $8 \%$ arginine and calcium carbonate. Am J Dent 2009;22 Spec No A:3A-7A

14 Yang ZY, Wang F, Lu K, Li YH, Zhou Z. Arginine-containing desensitizing toothpaste for the treatment of dentin hypersensitivity: a meta-analysis. Clin Cosmet Investig Dent 2016;8:1-14

15 Cunha-Cruz J, Stout JR, Heaton LJ, Wataha JC; Northwest PRECEDENT. Dentin hypersensitivity and oxalates: a systematic review. J Dent Res 2011;90(3):304-310

16 Saeki K, Marshall GW, Gansky SA, Parkinson CR, Marshall SJ. Strontium effects on root dentin tubule occlusion and nanomechanical properties. Dent Mater 2016;32(2):240-251

17 Babu KG, Subramaniam P, Teleti S. Remineralization potential of varnish containing casein phosphopeptides-amorphous calcium phosphate with fluoride and varnish containing only fluoride: a comparative study. Saudi J Oral Sci. 2018;5(1):35

18 Zalite V, Locs J. Characterization and Preparation of Calcium Phosphate Model Toothpaste for Tooth Enamel Remineralization. Key Eng Mater 2017;721:231:218

19 Kleinberg I. SensiStat. A new saliva-based composition for simple and effective treatment of dentinal sensitivity pain. Dent Today 2002;21(12):42-47
20 Strassler HE, Serio FG, Dentinal hypersensitivity: Etiology, Diagnosis And Management. The Academy of Dental Therapeutics and Stomatology; 2009:2-7

21 Arnold $\mathrm{WH}$, Prange M, Naumova EA. Effectiveness of various toothpastes on dentine tubule occlusion. J Dent 2015;43(4):440-449

22 Onwubu SC, Mdluli PS, Singh S, Tlapana T. A novel application of nano eggshell/titanium dioxide composite on occluding dentine tubules: an in vitro study. Braz Oral Res 2019;33:e016

23 Macri DV. Implementing a minimally invasive approach. Dimens Dent Hyg 2016;14:32-37

24 Haghgoo R, Mehran M, Ahmadvand M, Ahmadvand MJ. Remineralization effect of eggshell versus nano-hydroxyapatite on caries-like lesions in permanent teeth (in vitro) J Int Oral Health 2016;8:435:439.

25 Cutler ET, Prevention and treatment of oral diseases. In: Patent U, ed. USA: Squigle, Inc; 2014

26 Onwubu SC, Mdluli PS, Singh S, Nyembe S, Thakur R. An in situ evaluation of the protective effect of nano eggshell/ titanium dioxide against erosive acids. Int J Dent 2018. doi: $10.1155 / 2018 / 4216415$

27 Onwubu SC, Mdluli PS, Singh S. Evaluating the buffering and acid-resistant properties of eggshell-titanium dioxide composite against erosive acids. J Appl Biomater Funct Mater 2019;17(1):2280800018809914

28 Onwubu SC, Mdluli PS, Singh S, Lawrence M, Ngombane Y. Characterization and in vitro evaluation of an acid resistant nanosized dental eggshell-titanium dioxide material. Adv Powder Technol 2019;30(4):766-773

29 Saporeti MP, Mazzieiro ET, Sales WF. In vitro corrosion of metallic orthodontic brackets: influence of artificial saliva with and without fluorides. Dental Press J Orthod 2012;17(6):24e1-24e7

30 Cunha-Cruz J, Wataha JC, Heaton LJ, et al. Northwest Practice-based Research Collaborative in Evidence-based DENTistry. The prevalence of dentin hypersensitivity in general dental practices in the northwest United States. J Am Dent Assoc 2013;144(3):288-296

31 Hua Z, Nie M, Liu X, Wang Q. A clean strategy to prepare polylactide/hydroxyapatite bionanocomposites via solid mechanochemistry. J Macromol Sci, Part B. 2017;56(5):306-314

32 Fonseca RB, Haiter-Neto F, Fernandes-Neto AJ, Barbosa GAS, Soares CJ. Radiodensity of enamel and dentin of human, bovine and swine teeth. Arch Oral Biol 2004;49(11):919-922

33 Tanaka JLO, Medici Filho E, Salgado JAP, et al. Comparative analysis of human and bovine teeth: radiographic density. Braz Oral Res 2008;22(4):346-351

34 Yassen GH, Platt JA, Hara AT. Bovine teeth as substitute for human teeth in dental research: a review of literature. J Oral Sci 2011;53(3):273-282

35 Silva BG, Nunes Gouveia TH, Pereira da Silva MA, Bovi Ambrosano GM, Baggio Aguiar FH, Leite Lima DAN. Evaluation of home bleaching gel modified by different thickeners on the physical properties of enamel: an. in situ. study. Eur J Dent 2018;12(4):523-527

36 Vajrabhaya LO, Korsuwannawong S, Harnirattisai C, Teinchai C. Changes in the permeability and morphology of dentine surfaces after brushing with a Thai herbal toothpaste: a preliminary study. Eur J Dent 2016;10(2):239-244

37 Carvalho AO, Ayres AP, de Almeida LCAG, Briso ALF, Rueggeberg FA, Giannini M. Effect of peroxide bleaching on the biaxial flexural strength and modulus of bovine dentin. Eur J Dent 2015;9(2):246-250

38 Ayad F, Ayad N, Zhang YP, DeVizio W, Cummins D, Mateo LR. Comparing the efficacy in reducing dentin hypersensitivity of 
a new toothpaste containing $8.0 \%$ arginine, calcium carbonate, and $1450 \mathrm{ppm}$ fluoride to a commercial sensitive toothpaste containing $2 \%$ potassium ion: an eight-week clinical study on Canadian adults. J Clin Dent 2009;20(1):10-16

39 Docimo R, Montesani L, Maturo P, et al. Comparing the efficacy in reducing dentin hypersensitivity of a new toothpaste containing $8.0 \%$ arginine, calcium carbonate, and $1450 \mathrm{ppm}$ fluoride to a commercial sensitive toothpaste containing $2 \%$ potassium ion: an eight-week clinical study in Rome, Italy. J Clin Dent 2009;20(1):17-22

40 Nakashima S, Yoshie M, Sano H, Bahar A. Effect of a test dentifrice containing nano-sized calcium carbonate on remineralization of enamel lesions in vitro. J Oral Sci 2009;51(1):69-77

41 Chiang YC, Chen HJ, Liu HC, et al. A novel mesoporous biomaterial for treating dentin hypersensitivity. J Dent Res 2010;89(3):236-240

42 Yang H, Pei D, Chen Z, Lei J, Zhou L, Huang C. Effects of the application sequence of calcium-containing desensitising pastes during etch-and-rinse adhesive restoration. J Dent 2014;42(9):1115-1123

43 Yu J, Yang H, Li K, Lei J, Zhou L, Huang C. A novel application of nanohydroxyapatite/mesoporous silica biocomposite on treating dentin hypersensitivity: an in vitro study. J Dent 2016;50:21-29

44 Yang JC, Hu HT, Lee SY, et al. In vitro evaluation of dentin tubule occlusion for novel calcium lactate phosphate (CLP) paste. Materials (Basel) 2017;10(3):228

45 Wang Z, Sa Y, Sauro S, et al. Effect of desensitising toothpastes on dentinal tubule occlusion: a dentine permeability measurement and SEM in vitro study. J Dent 2010;38(5):400-410

46 Tao H, He Y, Zhao X. Preparation and characterization of calcium carbonate-titanium dioxide core-shell $\left(\mathrm{CaCO}_{3} @ \mathrm{TiO}_{2}\right)$ nanoparticles and application in the papermaking industry. Powder Technol 2015;283:308-314 